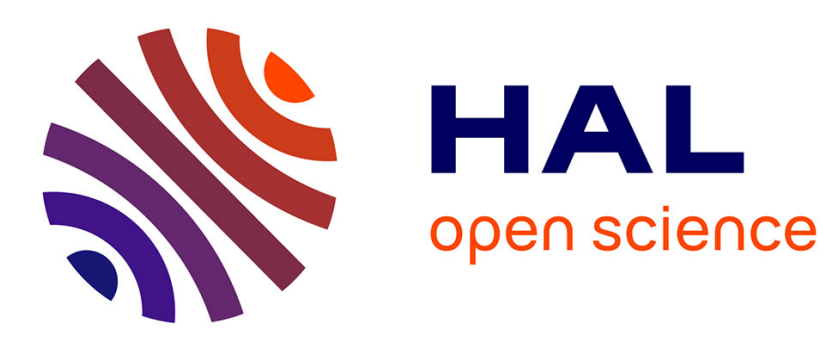

\title{
Signal processing correcting coupling between antennas in a $2 \times 2$ MIMO context
}

E. Graux, Nour Murad, David Carsenat, Bernard Jecko

\section{To cite this version:}

E. Graux, Nour Murad, David Carsenat, Bernard Jecko. Signal processing correcting coupling between antennas in a 2x2 MIMO context. First European Conference on Antennas and Propagation, 2006.

EuCAP 2006, Nov 2006, Nice, France. pp.1-6, 10.1109/EUCAP.2006.4584697 . hal-01221996

\section{HAL Id: hal-01221996 \\ https://hal.univ-reunion.fr/hal-01221996}

Submitted on 10 Nov 2017

HAL is a multi-disciplinary open access archive for the deposit and dissemination of scientific research documents, whether they are published or not. The documents may come from teaching and research institutions in France or abroad, or from public or private research centers.
L'archive ouverte pluridisciplinaire $\mathbf{H A L}$, est destinée au dépôt et à la diffusion de documents scientifiques de niveau recherche, publiés ou non, émanant des établissements d'enseignement et de recherche français ou étrangers, des laboratoires publics ou privés. 


\title{
SIGNAL PROCESSING CORRECTING COUPLING BETWEEN ANTENNAS IN A 2x2 MIMO CONTEXT
}

\author{
E. Graux, N. M. Murad, D. Carsenat and B. Jecko, Member, IEEE \\ $X L I M / O S A$ \\ 123, avenue Albert Thomas \\ 87060 LIMOGES Cedex, France \\ Emails: emmanuelle.graux@creape.unilim.fr, \{nour.murad,david.carsenat\}@xlim.fr
}

\begin{abstract}
The aim of this paper is to study a $2 \times 2$ Multiple-Input Multiple-Output (MIMO) with Alamouti technique and antenna's coupling phenomena at the transmitters and at the receivers. The motivation of this study is to show and to explain the impact of two different symbols estimation models over the coupling effect on the performances of the studied system. Some mathematical equations will demonstrate the behavior of the system and some curves will prove the results by simulations.
\end{abstract}

\section{INTRODUCTION}

MULTIPLE-INPUT MULTIPLE-OUTPUT (MIMO) wireless channels are known to offer better data rate and capacity by employing multiple antennas at both ends of the link [1]. An approach that benefits from the MIMO channel is the use of transmit diversity with space-time block-coding (STBC). Also, the use of multiple antennas at both ends of a wireless link has recently been shown to have the potential to drastically increase spectral efficiency through a technique known as spatial multiplexing (SM) [2-6]. In signal processing, the electromagnetic phenomena between two near antennas is seldom taken into account. Thus, in this paper, the study of phenomena is also undertaken, when the coherence length isn't respected. Those electromagnetic phenomena are called coupling, and their impact on the signal transmission are investigated here. Different estimations are studied too.

The outline of the paper is as follow: first, the electromagnetic phenomenon coupling with its notations is explained in section 2. In our model, analytical equations describe the system with Alamouti Technique [7] (section 3) for one particular symbols estimation, while the same study will be made in section 4 for another symbols estimation. Performance results are shown by simulations (section 5). To finish, analyses and conclusion are made in sections 6 and 7.

\section{COUPLING MODEL AND NOTATIONS}

Since this coupling's phenomenon is seldom taken into account in the studies, a 2x2 MIMO system with Alamouti
Technique is considered with coupling's phenomena between antennas at the transmitter and at the receiver sides. The coupling is defined as a part of one antenna's power which radiates on the other antenna [8]. In fact, a part of the energy over the first antenna is radiated on the second near antenna and vice versa.

Configurations between the transmit antennas and the receive antennas are different. In a wireless communication, the antennas at the Base Station (BS) and at the Mobile Station (MS) can't have the same configurations. This kind of link is used for fairly high flows (near 2Mbit/s).

In this article, a 2x2 MIMO system with Alamouti Technique is studied over a worst Rayleigh's fading channel.

In order to normalize the transmitted and received energy at antennas (Fig. 1), $\sqrt{\alpha}$ shows the part of energy which remains from transmit antenna $\mathrm{Tx}_{0}$ to itself and from transmit antenna $\mathrm{Tx}_{1}$ to itself. Also, $\sqrt{1-\alpha}$ is the part of energy which is radiated from transmit antenna $\mathrm{Tx}_{0}$ to transmit antenna $\mathrm{Tx}_{1}$ and which is reradiated by transmit antenna $\mathrm{Tx}_{1}$ and symmetrically from transmit antenna $\mathrm{Tx}_{1}$ to transmit antenna $\mathrm{Tx}_{0}$. The same model is made for the receiver part with $\beta$. In a realistic wireless communication system, it's not very probable to have exactly the same antennas at the emission and the reception parts. Moreover, antennas at the Base Station and at the Mobile Station (or a laptop) have different configurations and diverse geometrical designs, sensibility, directivity, gain, pattern radiations... Let's note $(\alpha, \beta) \in \mathbb{C}$ the couple of couplings coefficients respectively at the emission and at the reception. Also, $0 \leq\|\alpha\|^{2} \leq 1$ and $0 \leq\|\beta\|^{2} \leq 1$.

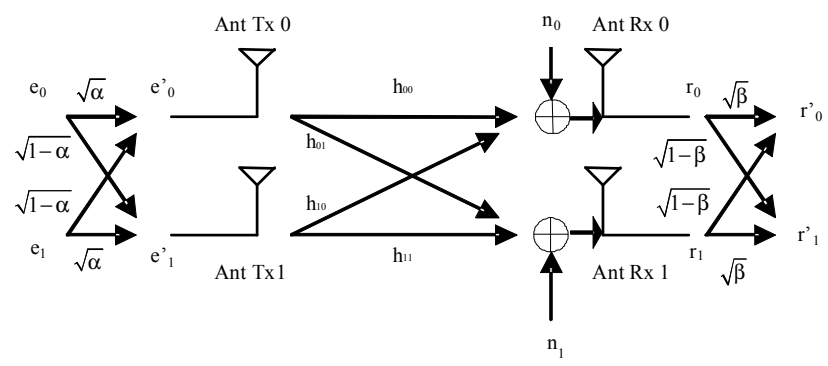

Figure 1. Coupling model.

These following notations are used along this paper:

- $T$ is the symbol time, 
- $e_{i}$ is the $i^{\text {th }}$ transmitted symbols sequence on the transmit antenna i with $i \in[0,1]$,

- $e_{i}^{\prime}$ is the $\mathrm{i}^{\text {th }}$ transmitted sequence on the transmit antenna $\mathrm{i}$ after coupling with $i \in[0,1]$,

- $h_{i j}$ is Rayleigh's fading coefficient from the $i^{\text {th }}$ transmit antenna to the $\mathrm{j}^{\text {th }}$ receive antenna. $h_{i j} \in \mathbb{C}$ and is quite constant during two symbol times $h_{i j}\left(t_{0}\right)=h_{i j}\left(t_{0}+T\right)$.

The impulse response of the channel is normalized as $\sum_{j=1}^{n_{T}}\left|h_{i j}\right|^{2}=n_{T}$ with $i, j \in \mathbb{N}, \mathrm{n}_{\mathrm{T}}$ the number of transmit antennas and $n_{R}$ the number of receive antennas [9], here $\mathrm{n}_{\mathrm{T}}=\mathrm{n}_{\mathrm{R}}=2$.

- $r_{i}$ is the received symbols sequence on the $i^{\text {th }}$ receive antenna,

- $r_{i}^{\prime}$ is the received symbols sequence on the $\mathrm{i}^{\text {th }}$ receive antenna after coupling,

- $n_{i}$ is Additive White Gaussian Noise (AWGN).

In this model, the Rayleigh channel and the couplings at each side correspond to the channel of this new model.

The cases $\|\alpha\|^{2}=0$ or $\|\beta\|^{2}=0$ mean that the antennas at the transmitter or at the receiver are coupled. In the other way, the cases $\|\alpha\|^{2}=1$ or $\|\beta\|^{2}=1$ mean that the antennas at each part of the communication link are uncoupled.

Two different estimations models are studied in this paper to equalize the received signal. The first one takes into account the totality of the received signal whereas the second one uses only one definite part of the received signal in order to reduce the quantity of noise. Even if the share of the used signal also decreases, the goal was to try to find a compromise.

\section{COUPLING ANALYSIS FOR ONE PARTICULAR SYMBOLS ESTIMATION MODEL}

In this section, analytical equations are written and describe precisely the couplings. The performance gains are analyzed. The symbols estimation model used in this section is the estimation with all terms (see eq. (7) for example).

Alamouti Technique [10] consists in transmitting coded symbols over the two transmit antennas. If the initial sequence of symbols is like than $\left[s_{0}, s_{1}, \ldots, s_{i}, \ldots\right]$, then $e_{0}=\left[s_{0},-s_{1}^{*}, s_{2},-s_{3}^{*}, \ldots, s_{2 i}, s_{2 i+1}^{*}, \ldots\right]$ and $e_{1}=\left[s_{1}, s_{0}^{*}, s_{3}, s_{2}^{*}, \ldots, s_{2 i+1}, s_{2 i}^{*}, \ldots\right]$ with $i \in \mathbb{N}$. The Alamouti's coder works on two consecutive symbol times. Then, the transmitted signals after coupling are

$$
\begin{gathered}
\left\{\begin{array}{l}
e_{0}^{\prime}\left(t_{0}\right)=\sqrt{\alpha} s_{0}+\sqrt{1-\alpha} s_{1} \\
e_{1}^{\prime}\left(t_{0}\right)=\sqrt{\alpha} s_{1}+\sqrt{1-\alpha} s_{0}
\end{array}\right. \\
\left\{\begin{array}{l}
e_{0}^{\prime}\left(t_{0}+T\right)=-\sqrt{\alpha} s_{1}^{*}+\sqrt{1-\alpha} s_{0}^{*} \\
e_{1}^{\prime}\left(t_{0}+T\right)=\sqrt{\alpha} s_{0}^{*}-\sqrt{1-\alpha} s_{1}^{*}
\end{array}\right.
\end{gathered}
$$

Next, before coupling the received signals are

$$
\begin{aligned}
& \left\{\begin{array}{l}
r_{0}\left(t_{0}\right)=s_{0}\left(h_{00} \sqrt{\alpha}+h_{10} \sqrt{1-\alpha}\right)+s_{1}\left(h_{00} \sqrt{1-\alpha}+h_{10} \sqrt{\alpha}\right)+n_{0} \\
r_{1}\left(t_{0}\right)=s_{0}\left(h_{01} \sqrt{\alpha}+h_{11} \sqrt{1-\alpha}\right)+s_{1}\left(h_{01} \sqrt{1-\alpha}+h_{11} \sqrt{\alpha}\right)+n_{1}
\end{array}\right. \\
& \left\{\begin{array}{l}
r_{0}\left(t_{0}+T\right)=s_{0}^{*}\left(h_{00} \sqrt{1-\alpha}+h_{10} \sqrt{\alpha}\right)-s_{1}^{*}\left(h_{00} \sqrt{\alpha}+h_{10} \sqrt{1-\alpha}\right)+n_{0} \\
r_{1}\left(t_{0}+T\right)=s_{0}^{*}\left(h_{01} \sqrt{1-\alpha}+h_{11} \sqrt{\alpha}\right)-s_{1}^{*}\left(h_{01} \sqrt{\alpha}+h_{11} \sqrt{1-\alpha}\right)+n_{1}
\end{array}\right.
\end{aligned}
$$

Afterward, the received signals after coupling are written as

$$
\begin{aligned}
& r_{0}^{\prime}\left(t_{0}\right)= s_{0}\left(\begin{array}{l}
h_{00} \sqrt{\alpha \beta}+h_{10} \sqrt{\beta(1-\alpha)}+ \\
h_{01} \sqrt{\alpha(1-\beta)}+h_{11} \sqrt{(1-\alpha)(1-\beta)}
\end{array}\right) \\
&+s_{1}\left(\begin{array}{l}
h_{00} \sqrt{(1-\alpha) \beta}+h_{10} \sqrt{\alpha \beta}+ \\
h_{01} \sqrt{(1-\alpha)(1-\beta)}+h_{11} \sqrt{\alpha(1-\beta)}
\end{array}\right)+\sqrt{\beta} n_{0}+\sqrt{1-\beta} n_{1} \\
& r_{1}^{\prime}\left(t_{0}\right)= s_{0}\left(\begin{array}{l}
h_{00} \sqrt{\alpha(1-\beta)}+h_{10} \sqrt{(1-\alpha)(1-\beta)}+ \\
h_{01} \sqrt{\alpha \beta}+h_{11} \sqrt{\beta(1-\alpha)}
\end{array}\right)+\sqrt{\beta} n_{1}+\sqrt{1-\beta} n_{0} \\
&+ s_{1}\left(\begin{array}{l}
h_{00} \sqrt{(1-\alpha)(1-\beta)}+h_{10} \sqrt{\alpha(1-\beta)}+ \\
h_{01} \sqrt{\beta(1-\alpha)}+h_{11} \sqrt{\alpha \beta}
\end{array}\right)+\sqrt{\beta} n_{1}+\sqrt{1-\beta} n_{0} \\
& r_{0}^{\prime}\left(t_{0}+\right.T)=s_{0}^{*}\left(\begin{array}{l}
h_{00} \sqrt{\beta(1-\alpha)}+h_{10} \sqrt{\alpha \beta}+ \\
h_{01} \sqrt{(1-\alpha)(1-\beta)}+h_{11} \sqrt{\alpha(1-\beta)}
\end{array}\right) \\
&-s_{1}^{*}\left(\begin{array}{l}
h_{00} \sqrt{\alpha \beta}+h_{10} \sqrt{\beta(1-\alpha)}+ \\
h_{01} \sqrt{\alpha(1-\beta)}+h_{11} \sqrt{(1-\alpha)(1-\beta)}
\end{array}\right)+\sqrt{\beta} n_{0}+\sqrt{1-\beta} n_{1} \\
& r_{1}^{\prime}\left(t_{0}+T\right)= \\
& s_{1}^{*}\left(\begin{array}{l}
h_{00} \sqrt{(1-\alpha)(1-\beta)}+h_{10} \sqrt{\alpha(1-\beta)} \\
+h_{01} \sqrt{\beta(1-\alpha)}+h_{11} \sqrt{\alpha \beta} \\
+h_{00} \sqrt{\alpha(1-\beta)}+h_{10} \sqrt{(1-\alpha)(1-\beta)}
\end{array}\right)
\end{aligned}
$$

Finally, the symbols are estimated thanks to the symbols estimation model where all the terms using $(\alpha, \beta)$ are taken into account: 


$$
\left\{\begin{aligned}
\tilde{s}_{0}= & {\left[\sqrt{\alpha \beta} h_{00}+\sqrt{\alpha(1-\beta)} h_{01}+\sqrt{\beta(1-\alpha)} h_{h_{0}}+\sqrt{(1-\alpha)(1-\beta)} h_{11}\right] r_{0}^{\prime}\left(t_{0}\right)+} \\
& {\left[\sqrt{\alpha(1-\beta)} h_{00}+\sqrt{\alpha \beta} h_{01}+\sqrt{(1-\alpha)(1-\beta)} h_{10}+\sqrt{\beta(1-\alpha)} h_{11}\right] r_{1}^{*}\left(t_{0}\right)+} \\
& {\left[\sqrt{\beta(1-\alpha)} h_{00}+\sqrt{(1-\alpha)(1-\beta)} h_{01}+\sqrt{\alpha \beta} h_{10}+\sqrt{\alpha(1-\beta)} h_{11}\right] r_{0}^{* *}\left(t_{0}+T\right)+} \\
& {\left[\sqrt{(1-\alpha)(1-\beta)} h_{00}+\sqrt{\beta(1-\alpha)} h_{01}+\sqrt{\alpha(1-\beta)} h_{00}+\sqrt{\alpha \beta} h_{11}\right] r_{1}^{* *}\left(t_{0}+T\right) } \\
\tilde{s}_{1}= & {\left[\sqrt{\beta(1-\alpha)} h_{00}+\sqrt{(1-\alpha)(1-\beta)} h_{01}+\sqrt{\alpha \beta} h_{10}+\sqrt{\alpha(1-\beta)} h_{11}\right] r_{0}^{*}\left(t_{0}\right)+} \\
& {\left[\sqrt{(1-\alpha)(1-\beta)} h_{00}+\sqrt{\beta(1-\alpha)} h_{01}+\sqrt{\alpha(1-\beta)} h_{10}+\sqrt{\alpha \beta} h_{11}\right] r_{1}^{\prime} r_{10}\left(t_{0}\right)-} \\
& {\left[\sqrt{\alpha \beta} h_{00}+\sqrt{\alpha(1-\beta)} h_{01}+\sqrt{\beta(1-\alpha)} h_{10}+\sqrt{(1-\alpha)(1-\beta)} h_{11}\right] r_{0}^{*}\left(t_{0}+T\right)-} \\
& {\left[\sqrt{\alpha(1-\beta)} h_{00}+\sqrt{\alpha \beta} h_{01}+\sqrt{(1-\alpha)(1-\beta)} h_{10}+\sqrt{\beta(1-\alpha)} h_{11}\right] r_{1}^{* *}\left(t_{0}+T\right) }
\end{aligned}\right.
$$

To study the behaviour of the diversity technique, we estimate the received SNR in computing the mean and the variance of the expression (7).

$$
\begin{gathered}
E\left[\tilde{s}_{0}\right]=0 \\
E\left[\tilde{s}_{1}\right]=0 \\
\sigma^{2}=E^{2}\left[\tilde{s}_{0}\right]=E\left[\tilde{s}_{0}^{2}\right]-E\left[\tilde{s}_{0}\right] \\
\sigma^{2}=E_{s}\left[\begin{array}{l}
\alpha^{2}\left(32 \beta^{2}-44 \beta-12\right)+ \\
\alpha\left(-29 \beta^{2}+41 \beta+12\right)- \\
28 \beta(1-\beta)+8
\end{array}\right] \\
+\frac{N_{0}}{2 \sqrt{2}}(4+(3 \alpha+13) \beta(1-\beta))
\end{gathered}
$$

The expression of the received SNR for the symbols is given by (10):

$$
\begin{aligned}
& S N R=\frac{E_{s}}{N_{0}} * 2 \sqrt{2} * \\
& {\left[\frac{\alpha^{2}\left(32 \beta^{2}-44 \beta-12\right)+\alpha\left(-29 \beta^{2}+41 \beta+12\right)-28 \beta(1-\beta)+8}{4+(3 \alpha+13) \beta(1-\beta)}\right]}
\end{aligned}
$$

The expression in square brackets in eq. (10) corresponds to the channel gain in this particular diversity technique.

Thanks to Eq. (10) it's easy to say that the received SNR (noted SNR) depends on three parameters: $\alpha, \beta$ and $\frac{E_{s}}{N_{0}}$, which is the transmitted SNR. The study of Eq. (10) gives some parametric curves. The parametric curves have been plotted for values of $\alpha$ which varies from 0 to 1 by step 0.1 . The more relevant curves are showed here.

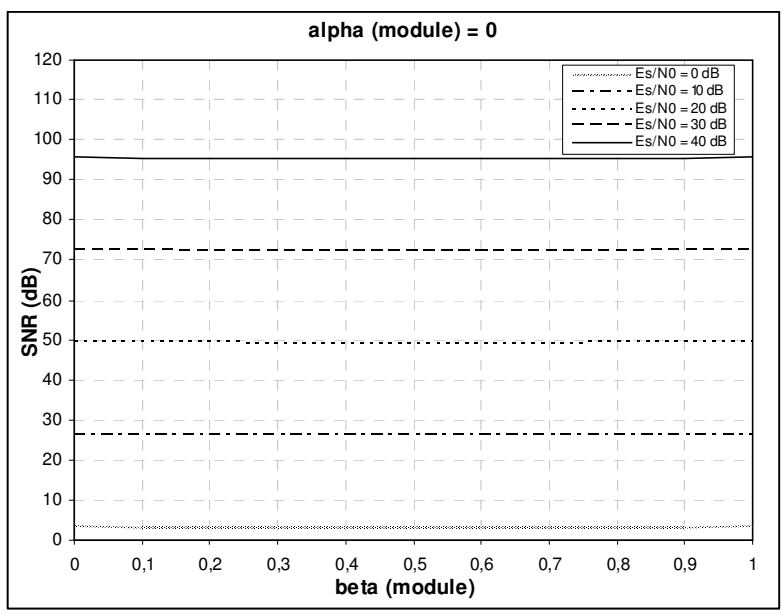

Figure 2. Analytic expressions' analysis for Alamouti Technique (first symbols estimation model), $\alpha=0$.

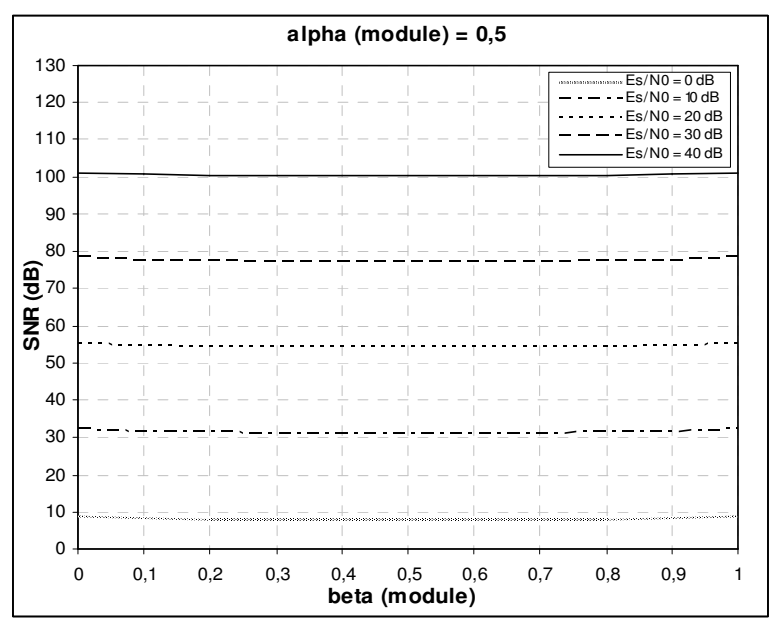

Figure 3. Analytic expressions' analysis for Alamouti Technique (first symbols estimation model), $\alpha=0.5$.

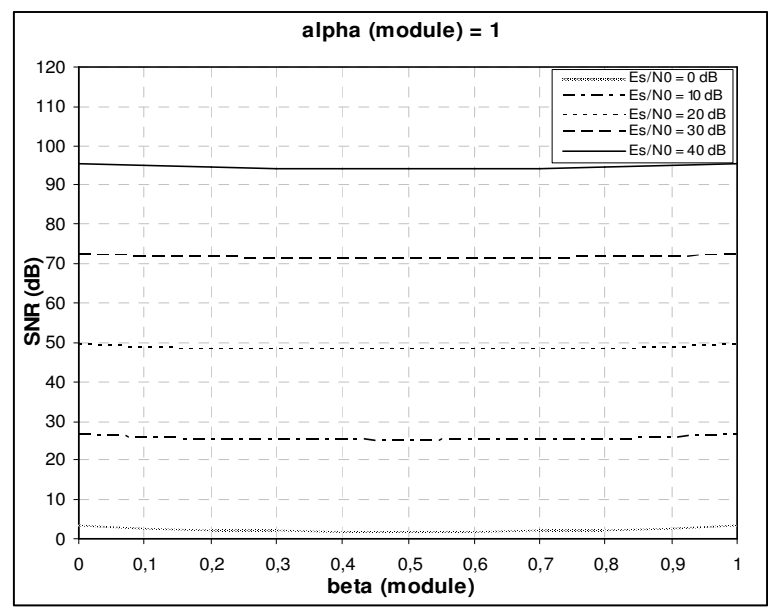

Figure 4. Analytic expressions' analysis for Alamouti Technique (first symbols estimation model), $\alpha=1$. 
These curves show that the more the ratio $\frac{E_{s}}{N_{0}}$ is high, the more the performances are good, what is logical because the receives $\mathrm{SNR}$ is a linear positive increasing function of $\frac{E_{s}}{N_{0}}$. The optimal couple of couplings is $\left(\alpha_{\text {opt }}, \beta_{\text {opt }}\right)=(0.5,1)$ (in module, the phase is equal to zero). So, to have the best performances, the power transmission must be shared in two equal parts on the two transmit antennas.

But for $\beta=1$ and an unspecified value of $\alpha$ and $\frac{E_{s}}{N_{0}}$, the SNR doesn't enormously vary, so with numerical simulations, the performances of the system will be closed whatever the value of $\alpha$. Here, with numerical values, it's easy to say that a particular values of $\alpha$ or $\beta$ will give the best performances, but with numerical simulations, the difference will not be also obvious.

\section{COUPLING ANALYSIS FOR ANOTHER SYMBOLS ESTIMATION MODEL}

In this part, the estimation of symbols is different from the one used in section 3. The goal was to find a new estimation of the symbols which allows reducing the noise quantity. In this section, the estimation will take into account only the terms which contain $\sqrt{\beta}$. By the means of this new symbols estimation model, the loss of signal is compensated by the reduction in the quantity of noise.

The equations which describe the studies system are the same that in section 3, but the estimation of the symbols is different. In eq. (7), only the terms which contains $\sqrt{\beta}$ are preserved. So, in this section, the symbols are estimated thanks to eq. (11):

$$
\left\{\begin{aligned}
\tilde{s}_{0}= & {\left[\sqrt{\alpha \beta} h_{00}+\sqrt{\beta(1-\alpha)} h_{10}\right]^{*} r_{0}^{\prime}\left(t_{0}\right)+\left[\sqrt{\alpha \beta} h_{01}+\sqrt{\beta(1-\alpha)} h_{11}\right]^{*} r_{1}^{\prime}\left(t_{0}\right)+} \\
& {\left[\sqrt{\beta(1-\alpha)} h_{00}+\sqrt{\alpha \beta} h_{10}\right] r_{0}^{*}\left(t_{0}+T\right)+\left[\sqrt{\beta(1-\alpha)} h_{01}+\sqrt{\alpha \beta} h_{11}\right] r_{1}^{* *}\left(t_{0}+T\right) } \\
\tilde{s}_{1}= & {\left[\sqrt{\beta(1-\alpha)} h_{00}+\sqrt{\alpha \beta} h_{10}\right] r_{0}^{\prime}\left(t_{0}\right)+\left[\sqrt{\beta(1-\alpha)} h_{01}+\sqrt{\alpha \beta} h_{11}\right]^{*} r_{1}^{\prime}\left(t_{0}\right)-} \\
& {\left[\sqrt{\alpha \beta} h_{00}+\sqrt{\beta(1-\alpha)} h_{10}\right] r_{0}^{*}\left(t_{0}+T\right)-\left[\sqrt{\alpha \beta} h_{01}+\sqrt{\beta(1-\alpha)} h_{11}\right] r_{1}^{* *}\left(t_{0}+T\right) }
\end{aligned}\right.
$$

And so, the expression of the SNR becomes:

$$
S N R=\frac{E_{s}}{N_{0}} \frac{\sqrt{2}}{4} *\left[\begin{array}{l}
23 \alpha(1-\alpha)-\sqrt{\alpha(1-\alpha)}(\beta+3)+ \\
\beta\left(20.5 \alpha^{2}-6.5 \alpha+15\right)+1
\end{array}\right]
$$

As in the last sub-section, it will be possible to study the behavior of this SNR with the three parameters: $\alpha, \beta$ and
$\frac{E_{s}}{N_{0}}$. The expression: $\left[\begin{array}{l}23 \alpha(1-\alpha)-\sqrt{\alpha(1-\alpha)}(\beta+3)+ \\ \beta\left(20.5 \alpha^{2}-6.5 \alpha+15\right)+1\end{array}\right]$ represents the channel gain.

Thanks to eq. (12), it's easy to say that the received SNR again depends on three parameters: $\alpha, \beta$ and $\frac{E_{s}}{N_{0}}$.

The study of eq. (12) allows plotting the following curves:

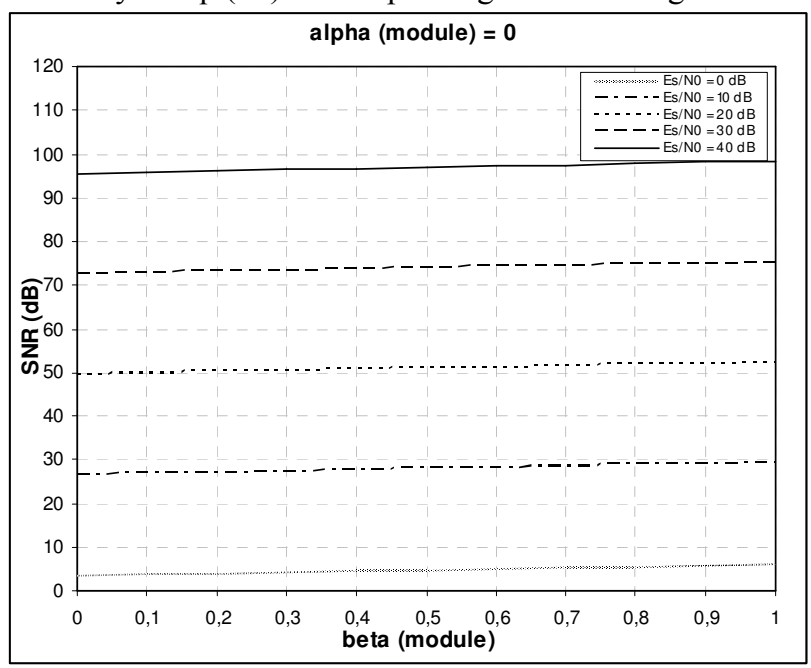

Figure 5. Analytic expressions' analysis for Alamouti Technique (second symbols estimation model), $\alpha=0$.

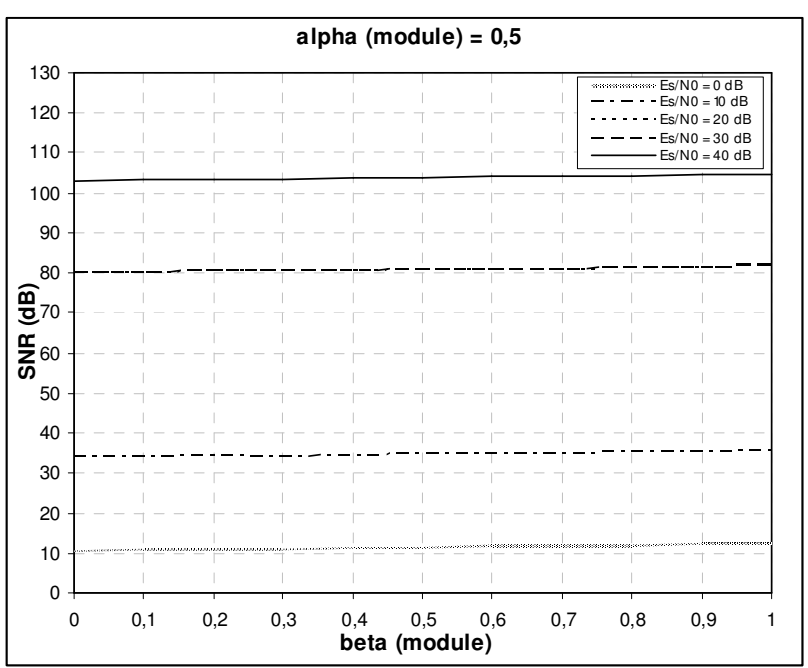

Figure 6. Analytic expressions' analysis for Alamouti Technique (second symbols estimation model), $\alpha=0.5$. 


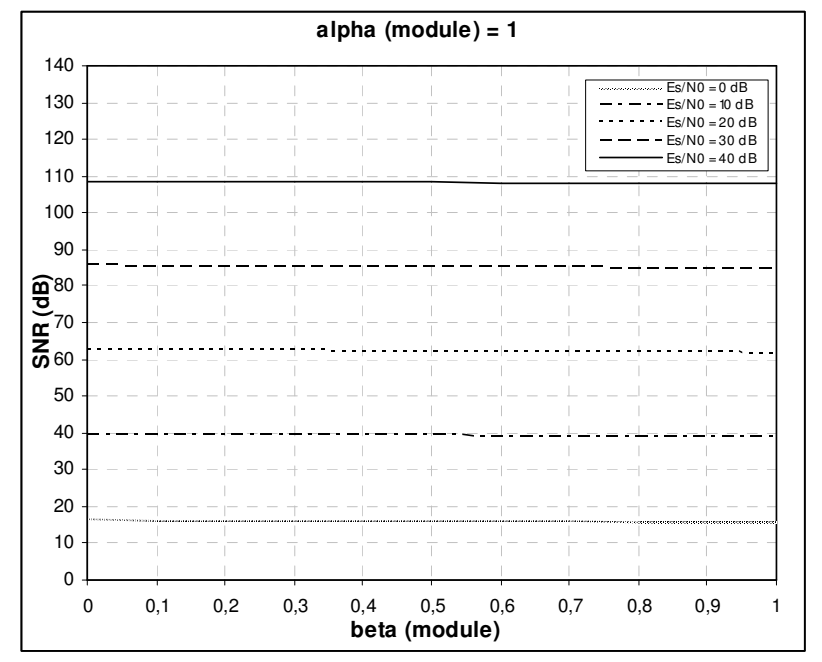

Figure 7. Analytic expressions' analysis for Alamouti Technique (second symbols estimation model), $\alpha=1$.

The optimal values of the couplings are $\left(\alpha_{\text {opt }}, \beta_{\text {opt }}\right)=(1,(0$ or 1$))$. The results are very closed, so it's undeniable that $\beta_{\text {opt }}=1$ is the optimal value, but for $\alpha$ it's not also obvious. Here, the optimal values found show that the best performances will be obtained for a system with no coupling effect at each side of the transmission system.

\section{NUMERICAL SIMULATION RESULTS}

In this section, realistic simulations are made for the two estimated models in a numerical wireless communication chain.

\subsection{First symbols estimation model}

Thanks to a simulation plan, the optimal values of the couplings for the studied system have been found.

The coupling coefficients are complex numbers written as:

$$
\begin{aligned}
& \alpha=\rho e^{j \theta} \\
& \beta=\rho e^{j \theta^{\prime}}
\end{aligned}
$$

The optimal coupling coefficients are:

$$
\left\{\begin{array}{l}
\alpha_{o p t}=0.5 e^{j 0}=0.5 \\
\beta_{o p t}=1 e^{j 0}=1
\end{array}\right.
$$

These values show that it's more judicious to have signals in phase.

For analysis, to obtain a BER of $10^{-6}$, the system with no diversity needs $\frac{E_{s}}{N_{0}} \simeq 57 d B$ (SNR at the emission part), whereas Alamouti Technique needs $\frac{E_{s}}{N_{0}} \simeq 17.8 d B$. The profit is near $40 \mathrm{~dB}$ to reach the same quality of service.

\subsection{Second symbols estimation model}

In this part, a second estimation model is studied. For the three techniques described in the first section, some numerical simulations made it possible to find the optimal coupling coefficients. The best performances are obtained when the coupling coefficients are equal to:

$$
\left\{\begin{array}{l}
\alpha_{\text {opt }}=1 e^{j 0}=1 \\
\beta_{\text {opt }}=1 e^{j 0}=1
\end{array}\right.
$$

The results show that for this particular estimation model, the best performance will be obtained when there're no couplings at each part of the communication link.

For this second symbols estimation model, the optimal results are generally obtained for $\alpha_{o p t}=1$ or $\beta_{o p t}=1$, this means that the best results are for an uncoupled antennas system. The curves can also give to the users the behaviour of the communication link if the coupling coefficients change and deviate from their optimal value. For the studied system, to obtain a BER of $10^{-6}$, the required SNR at the emission part may be $\frac{E_{s}}{N_{0}} \simeq 12 d B$.

\section{ANALYTICAL AND NUMERICAL SIMULATION COMPARISON}

Initially, a comparison between the performances with optimal couplings for the two diverse symbols estimations can be made (Fig. 18).

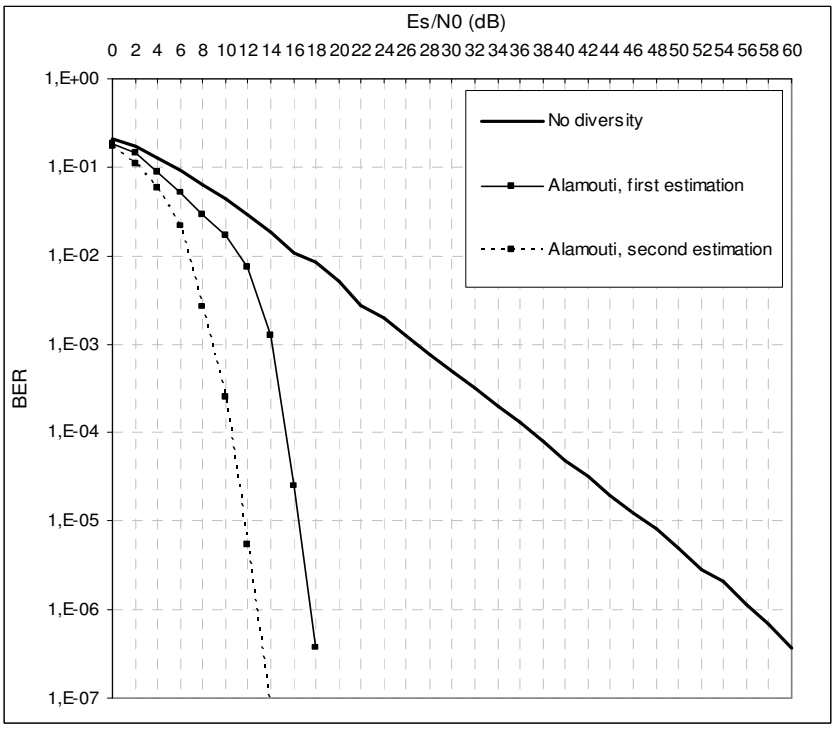

Figure 8. Comparison of the results for each symbols estimation model.

Figure 8 shows that performances vary according to the 
selected symbols estimation model. For Alamouti Technique, the second symbols estimation allows better results than the first one. For example, to obtain a BER of $10^{-6}$, the system with the first symbols estimation needs $\frac{E_{s}}{N_{0}} \simeq 17.8 d B$ and the same system with the second symbols estimation needs $\frac{E_{s}}{N_{0}} \simeq 12.8 d B$. This reduces a little the necessary transmit SNR to reach this particular BER.

The results are gathered in the two following tables:

\begin{tabular}{|l|l|l|l|}
\hline & $\begin{array}{c}\text { Alamouti } \\
\text { Technique }\end{array}$ & $\begin{array}{c}\text { No } \\
\text { diversity }\end{array}$ & Profit \\
\hline $\mathrm{BER}=10^{-3}$ & $14.1 \mathrm{~dB}$ & $26.8 \mathrm{~dB}$ & $12.7 \mathrm{~dB}$ \\
\hline $\mathrm{BER}=10^{-6}$ & $17.8 \mathrm{~dB}$ & $56.6 \mathrm{~dB}$ & $38.8 \mathrm{~dB}$ \\
\hline
\end{tabular}

Table 1. Comparison (first symbols estimation model).

\begin{tabular}{|l|l|l|l|}
\hline & $\begin{array}{c}\text { Alamouti } \\
\text { Technique }\end{array}$ & $\begin{array}{c}\text { No } \\
\text { diversity }\end{array}$ & \multicolumn{1}{|c|}{ Profit } \\
\hline $\mathrm{BER}=10^{-3}$ & $9 \mathrm{~dB}$ & $26.8 \mathrm{~dB}$ & $17.8 \mathrm{~dB}$ \\
\hline $\mathrm{BER}=10^{-6}$ & $12.8 \mathrm{~dB}$ & $56.6 \mathrm{~dB}$ & $43.8 \mathrm{~dB}$ \\
\hline
\end{tabular}

Table 2. Comparison (second symbols estimation model).

Then, an analysis is carried on to release the similarities between the analytical expressions and the results of numerical simulations. In fact, when the transmit SNR increases, the performances are better, so the BER decreases and vice versa.

The analysis of the SNR expressions enables us to understand the behaviour of the studied system according to the various parameters: $\alpha, \beta$ and $\frac{E_{s}}{N_{0}}$. In this studied case, the expression of the SNR can be studied for one parameter by fixing the other parameters.

If the function is studied with the parameter $\frac{E_{s}}{N_{0}}$, the function is linear, it's to say that the higher $\frac{E_{s}}{N_{0}}$ is, the more the received SNR is elevated. If the expression is now studied according to the parameters $\alpha$ and $\beta$, the analysis shows that the analytical study and the numerical simulations coincide for the two symbols estimation models.

\section{CONCLUSION}

Between theoretical curves (obtained thanks to the study of eqs. (10) and (12)) and optimal couplings coefficients $\left(\alpha_{o p t}, \beta_{\text {opt }}\right)$ found with the numerical simulations, the results are closed.

The optimal couplings are often equal to one (in module).
This corresponds in fact to no coupling between the antennas. So, the coupling between antennas often degrades the performances of the connection link. According to the studied technique, the two diverse estimations don't give the same results and offer solutions to equalize the received signal with the a priori knowledge of the coupling coefficients. The analytical study permits to know how the diversity system will behave according to the various parameters. With the numerical simulations, it's easy to know if a value of coupling will considerably degrade the performance of the system or if the degradation will tiny. In this study, the antennas at the transmit or receive sides are much closed, so the channels will be correlated.

\section{REFERENCES}

1. I.E. Telatar "Capacity of multi-antenna Gaussian Channel", European Transactions of Telecommunications, 10(6):585-595, 1999.

2. G.J. Foschini and M.J. Gans "On limits of wireless communications in a fading environment when using multiple antennas", Wireless Personal Communications, 6(3):311-335, 1998 .

3. T. Svantesson, "A physical MIMO radio channel model for multi-element multi-polarized antenna systems", Vehicular Technology Conference, VTC 2001, Fall. IEEE VTS 54th, Vol.2, pp. $1083-1087$.

4. Kyritsi P. and Cox D.C., "Effect of element polarization on the capacity of a MIMO system", IEEE Wireless Communications and Networking Conference, WCNC 2002, Vol. 2, pp. 892 - 896.

5. Baum D.S., Gore D., Nabar R., Panchanathan S., Hari K.V.S., Erceg V. and Paulraj A.J. , "Measurement and characterization of broadband MIMO fixed wireless channels at $2.5 \mathrm{GHz}$,' IEEE International Conference on Personal Wireless Communications, 2000, pp.: 203 -206.

6. R.U. Nabar, H. Bölcskei, V. Erceg, D. Gesbert and A.J. Paulraj, ., "Performance of multiantenna signaling techniques in the presence of polarization diversity", IEEE Transactions on Signal Processing, vol. 50, No. 10, October 2002.

7. C. Degen and W. Keusgen, "Performance evaluation of MIMO systems using dual-polarized antennas", 10th International Conference on Telecommunications, ICT 2003, Vol.2, pp. $1520-1525$.

8. J. J. Carr, Practical Antenna Handbook, McGraw-Hill, 4 edition, May 23, 2001, ISBN: 0071374353.

9. B. Vucetic, J. Yuan, Space-Time Coding, Wiley, 2004, ISBN : 0470847573.

10. S.M. Alamouti, "A Simple Transmit Diversity Technique for Wireless Communications", IEEE Journal on Selected Areas on Communications, 16(8):1451-1458, 1998. 